\title{
LA INFORMACIÓN Y SUS RELACIONES
}

\section{Caracterización de la información}

En el conjunto de movimientos y transformaciones que ocurren en el universo, se destaca con claridad la conexión recíproca existente entre todos y cada uno de los procesos. Tales conexiones son múltiples, polimorfas y activas. La influencia mutua se ejerce tanto directamente, por contigüidad, como de una manera indirecta, por mediación de otros procesos. En cuanto a su forma, dicha influencia adopta diversas modalidades. $Y$ una de esas modalidades es la información que los procesos pueden transmitir a otros procesos o, bien, recibir de ellos, respecto a los cambios que se producen respectivamente en su seno.

El hombre adquiere información acerca de los procesos con los cuales entra en relación, a través de las acciones espontáneas que lo afectan y por medio de las experiencias que realiza continuamente. A la vez, con su actividad práctica, el hombre transmite a los procesos exteriores la información requerida para efectuar en ellos los cambios que se propone. Asf, el intercambio de información es parte integrante de las actividades que permiten al hombre adaptarse al medio ambiente y, simultáneamenie, transformarlo de acuerdo con sus necesidades. Por otra parte, la informarión es la forma elemental del conocimiento; y su acumulación, la recolección de datos, constituye la materia prima con la cual se elabora el conocimimto científico.

La información que el hombre adquiere es coordinłda por su sistema nervioso y sometida a una serie de procesos complejos en su cerebro -en los cuales interviene también la información acumulda previamente, lo mismo que los resultados elaborados y probados con anterioridad-, hasta que finalmente su efecto y su consecuencia emerge a través de sus órganos efectores en la forma de una acción, la cual lleva aparejada la transmisión de otra información diferente a la recibida. Por otro lad, el cuerpo humano en su conjunto, comprendiendo a todas y cada una de suc células, moléculas y átomos, se encuentra organizado activamente por diveras vínculos que posee para la adquisición, el empleo, la acumulación, la transmison y y la transferencia de información, tanto hacia el medio ambiente exterior internamente. 1

1 Véase, Y. P. Frolov, La cibernética actual y el cerebro humano, Suplementos del 1 Véase, Y. P. Frolov, La cibernética actual y el cerebro humano, Suplemesticos, Segunda Serie, núm.. 13, Universidad
Seminario de Problemas Cientificos y Filosóficos, Nacional de México, $195^{8 .}$ 


\section{Aspectos cualitativos de la información}

La información es un conjunto de señales, mensajes, comunicaciones, permisiones, prohibiciones, órdenes, estímulos o impulsos de cualquiera índole, con los que un proceso manifiesta su existencia y provoca cierta perturbación en otros procesos, la cual puede dar lugar a la ejecución de acciones determinadas. El carácter de la información es sumamente variado y depende naturalmente de la clase de proceso de la cual proviene y de las modalidades de su existencia. La comunicación de la información se efectúa en forma de una transmisión o de una transferencia. $Y$ los efectos provocados por la información en los procesos que la reciben, también dependen de las características de éstos y pueden ser inmediatos o mediatos, directos 0 indirectos. ${ }^{2}$

Toda información puede ser considerada desde dos puntos de vista distintos: en cuanto a su calidad o atendiendo a su cantidad. ${ }^{3}$ Cualitativamente, cada información representa un conjunto de propiedades diversas y es susceptible de ser utilizada de diferentes maneras. En todo caso, esas propiedades y maneras se descubren históricamente en el curso del desarrollo social.4 Otro tanto sucede con la invención de los procedimientos adoptados para estimar el contenido cualitativo de la información. Algunas veces, la estimación que re concede a una información corresponde a su naturaleza, otras veces está determinada por las condiciones o circunstancias en que se adquiere, y en oras ocasiones obedece sencillamente a una convención.

Otro aspecto culitativo de la información es el valor o la importancia que se le atribuye c que efectivamente representa para quien la recibe.5 En estrecha relación cor el significado de la información, se encuentra el es-

2 Para profundizar et el concepto de información, además de las obras a que luego hacemos referencia, se pueden consultas también las siguientes: $L$. de Broglie, ed., La cybernetique, théorie du signal et de l'information, París, Editions de la Revue d'Optique, 1951; M. Bunge, "Do Computers Think)", The British Journal for the Philosophy of Science, vol. VII, núms. 26 y 27, 1956; S. Goldman, Information Theory, Londres, Constable, 1953; W. Jackson, ex., Communication Theory, Londres, Butterworth, 1952; J. Piaget, W. Mays y W. Beth, Psiologia, logica y comunicación, Buenos Aires, Nueva Visión, 1959; J. R. Pierce, Simbolos, snales y ruidos, Revista de Occidente, Madrid, 1962; H. Rodriguez, Psicologia y ciber-etia, Siglo Veinte, Buenos Aires, 1958; P. M. Woodward, Probability and Informatir Theory, with Application to Radar, Londres, Pergamon Press, 1953.

3 Véaś- C. Marx El capital, México, Fondo de Cultura Económica, 1959, vol. I, pág. 3.

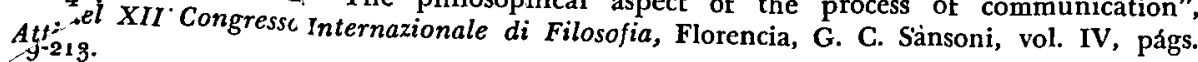

5 Léon Brillouin, er Science and Information Theory, Nueva York, Academic Press,
1956, págs. 10, 294 y 297, considera alcance de la teoria; Problems of Cybernetics, en cambio, A. A. Kharkevitz, en "On the value of information", cuantificación del valor de cremento de la probabilidad información, estableciendo su medida en función del in- 
tablecimiento de criterios adecuados para seleccionar la información requerida en cada caso. Una vez adquirida la información, se plantea el problema de investigar los procesos a que es sometida, tanto en las máquinas calculadoras como en los servomecanismos y otros dispositivos de automatización. Igualmente, se impone la necesidad de estudiar con rigor los procesos del pensamiento, que se efectúan en el cerebro humano a partir de la información adquirida y con base en los procedimientos elaborados con las informaciones anteriores. ${ }^{6}$

\section{La cantidad de información}

Entre la diversidad de procesos objetivos con cualidades tan diferentes, en los cuales se emite o se absorbe información de muchas maneras, existen relaciones cuantitativas que son análogas y comunes a todos ellos. Tales relaciones cuantitativas han permitido establecer una unidad de medida, eligiendo para eso una magnitud tomada directamente de la cuantificación de los mismos procesos objetivos de información y reduciéndola a su expresión elemental y constante. Una vez determinada la unidad de información, ha sido posible determinar los procedimientos de medición de la información, que son otras tantas maneras de contar o de calcular el número de veces que dicha unidad de medida se encuentra comprendida en una información, para fijar así precisamente su cantidad.

En cada proceso existe un cierto número de cambios de estado que son posibles. Cuando todavía no se obtiene información a.guna acerca de un proceso, todos esos posibles cambios de estado tienen la visma probabilidad de realizarse. Pero, a medida que se obtiene intormación sobre el proceso en cuestión, se reduce el número de posibilidades por la exclusión de aquellos cambios de estado que se van realizando. Hasta que linalmente, cuando se llega a obtener una información completa, entonces d número de posibilidades queda reducido a cero. ${ }^{7}$

Por lo tanto, la cantidad de información $I$ contènida en un proceso, se puede expresar como una función logarítmica del nịmero de cambios de estado $P$ del proceso, que son posibles e igualmente prohables; introduciendo al propio tiempo una constante de proporcionalidad $K$, qus permite sumar las informaciones obtenidas en condiciones independientes. ${ }^{8}$ As. la cantidad de información queda expresada por la ecuación:

$$
I=K \log P \text {. }
$$

6 Frolov, op. cit.

7 Véase W. Ross Ashley, Introducción a la cibernética, Bıenos Aires, Nueva Visión, 1960, págs. 14 y 171 .

8 Brillouin, op. cit., págs. 1-3. 
Ahora bien, cada uno de los posibles cambios de estado de un proceso se encuentra sujeto, en rigor, a la alternativa dilemática de realizarse o de no realizarse. Esa disyuntiva constante, representa la cantidad elemental de información que se puede obtener sobre un proceso, o sea, que toda información es susceptible de descomponerse en un número entero de esas cantidades elementales. Por consiguiente, su magnitud ha sido elegida como la unidad de información. Para ello se utiliza el sistema binario de los dígitos $I$ y $o$, referido a la realización o a la falta de realización de un cambio de estado, dentro del conjunto de cambios de estado que son posibles, igualmente probables y mutuamente excluyentes. Entonces, la unidad de información es el ardite ${ }^{9}$ que representa la cantidad de información contenida en una posibilidad dilemática que se resuelve necesariamente en $\mathrm{Si}$ o $\mathrm{No}$, o bien, en sus valores numéricos correspondientes $I$ y $o$.

Con la adopción del ardite como unidad de información, el número de posibilidades de cambio existentes en un proceso determinado, resulta ser:

$$
P=2^{n} .
$$

Sustituyendo este valor de $P$ en la ecuación de la información antes establecida, tenemos:

$$
I=K \log P=K \log 2^{n}=K n \log 2 ；
$$

luego, identificando la cantidad de información $I$ con el número de ardites de información, $n$, resulta:

$$
K=\frac{1}{\log 2}=\log _{2} e ;
$$

con lo cual, la cantidad de información medida en ardites, queda expresada finalmente por la ecuación:

$$
I=\log _{2} P .
$$

Es interesante hacer notar que el ardite, como cantidad mínima de información que es posible obtener acerca de un proceso, viene a ser al propio tiempo el cuanto de información; ya que es la dosis más pequeña que puede darse y, a la vez, porque cualquier cantidad de información mayor es siempre un múltiplo entero de ella. Por ende, el valor del ardite es enteramente objetivo e independiente del observador. Asimismo, la cantidad de información medida en ardites tiene siempre el mismo valor numérico para todos los observadores..$^{10}$

9 Es la unidad binaria conocida también con los nombres de bit y de hartley.

10 Brillouin, op. cit., pág. 10. 


\section{Propiedades termodinámicas}

La primera ley de la termodinámica expresa que en todos los procesos, lo mismo que en los cambios de estado que ocurren en ellos, existe una propiedad denominada energía que representa la capacidad para producir efectos. ${ }^{11}$ La energía tiene la peculiaridad de que su cantidad total se conserva constante, aun cuando se presenta en varias formas diferentes y en cada una de ellas es susceptible de transformarse en cualquiera de las otras, conforme a una equivalencia bien determinada. Además, como tambiẻn es posible la conversión mutua entre la masa y la energía, en la proporción indicada por la conocida ecuación de Einstein: $E=m c^{2}$; tenemos que la energía es capaz de transferirse y manifestarse en forma de masa y, viceversa, la masa en cualquiera de las formas de energía.

La segunda ley de la termodinámica establece la existencia de otra propiedad común a los cambios de estado de los procesos, conocida con el nombre de entropia, la cual puede ser considerada como la tendencia hacia la desaparición de las diferencias de temperatura, que es el estado más probable entre todos los posibles. ${ }^{12} \mathrm{La}$ entropia se incrementa con los cambios de estado que son irreversibles, y permanece constante en aquellos cambios que son reversibles. El incremento de la entropia no afecta la constancia cuantitativa de la energía, pero sí influye en su capacidad para ejecutar trabajo.13 Mientras subsisten diferencias de temperatura, es posible que la energía produzca como efecto la ejecución de trabajo; en tanto que esa posibilidad desaparece cuando se alcanza el equilibrio termodinámico. Por ello es que la entropia puede ser interpretada como la sombra de la energia. ${ }^{14}$

La tercera ley de la termodinámica enuncia que, cuando disminuye indefinidamente la temperatura de un proceso químicamente homogéneo y de densidad finita, entonces su entropia se aproxima indefinidamente a un valor definido, que es independiente de la presión, del estado de cohesión y de los cambios quimicos particulares. Como la expresión del valor de la entropia contiene una constante arbitraria, resulta que en el límite, en el cero absoluto de temperatura, la entropia de un proceso es igual a cero. $\mathrm{Y}$ esta conclusión teórica se encuentra de acuerdo con los resultados obtenidos en la experimentación. ${ }^{15}$

11 M. Planck, General Mechanics, Londres, Macmillan, 1933, pág. $7^{6 .}$

12 M. Planck, Treatise on Thermodynamics, Nueva York, Dover, 1945, pág. 106.

13 M. Planck, Theory of Heat, Londres, Macmillan, 1932, págs. 54-55.

14 Véase, E. Kolman, Qué es la cibernética, Suplementos del Seminario de Problemas Científicos y Filosóficos, Segunda Serie, Núm. 19, Universidad Nacional de México, 1958. 15 Planck, Treatise on Thermodynamics, págs. 273-274. 


\section{Los vinculos entre la información y la entropia}

El crecimiento de la entropia significa que el proceso se aproxima al estado de equilibrio termodinámico, que es a la vez el estado más probable, porque puede realizarse en un número mayor de maneras diferentes. Entonces, la entropia se encuentra vinculada directamente con la probabilidad; y ambas tienden naturalmente a incrementarse en una proporción logarítmica. Su relación queda expresada por la ecuación de Boltzmann-Planck:

$$
S=k \log P,
$$

en donde $S$ es la entropia del proceso, $k$ la constante de Boltzmann y $P$ la probabilidad termodinámica, o sea, el número total de estados elementales del proceso. De esta manera, la entropia es la medida de la probabilidad de que un proceso se encuentre en un estado determinado.16

Entre la entropia y la información existe una conexión inmediata. ${ }^{17}$ La entropia es una medida de la carencia de información acerca de un proceso, porque representa la cantidad de información que falta por obtener. ${ }^{18}$ En efecto, cuando no se ha obtenido ninguna información sobre un proceso, se tiene el mayor número de posibilidades y un máximo de entropia. Cuando se obtiene información, al mismo tiempo que se restringe el número de posibilidades, decrece la entropia del proceso. ${ }^{19}$ Al obtenerse la información completa o máxima, una de las posibilidades adquiere una probabilidad igual a la unidad - es decir, que se convierte en certeza-, en tanto que se anulan las probabilidades de todas las otras posibilidades y el valor de la entropia se reduce a un mínimo.

En el sistema cgs, la unidad de medida para la entropia tiene las dimensiones de la energía dividida entre la temperatura. Así, el valor de la constante de Boltzmann es:

$$
k=1.3^{8} \times 10^{-16} \text { ergios por grado centígrado. }
$$

Tomando en cuenta la relación existente entre la entropia y la información, se puede utilizar esta constante $k$, en lugar de la constante $K$ de la ecuación de la información, para medir la cantidad de información en unidades de entropia. También se puede medir la entropia en unidades de información. ${ }^{20}$ En tal caso, la relación entre las unidades de los dos sistemas es:

16 Planck, Theory of Heat, págs. 226-228.

17 La semejanza entre la información y la entropia fue advertida por L. Szilard, Zeitschrift für Physik, 53, 1929; y su conexión fue descubierta por C. Shannon y W. Weaver, The Mathematical Theory of Communication, Urbana, University of Illinois Press, 1949. 18 Brillouin, op. cit., pág. xii.

19 Véase, G. T. Guilbaud, What is Cybernetics?, Nueva York, Grove Press, 1960, pág. 107.

20 Brillouin, op. cit., pág. \$. 


$$
\frac{k}{K}=1.3^{8} \times 10^{-16} \log 2=0.9565 \times 10^{-16} \approx 10^{-16} \text {. }
$$

Como se puede advertir fácilmente, la contribución que resulta en la práctica para la entropia de un proceso, es una cantidad tan pequeña que es. despreciable en comparación con la entropia total. Así, por ejemplo, en una red telefónica que tenga 100000000 de suscriptores, como cada uno de ellos tiene la posibilidad de ser comunicado con cualquiera de los otros, tenemos:

$$
P=n^{n},
$$

siendo: $n=100000000=10^{8}$.

Entonces, la cantidad de información de esa red telefónica será:

$$
I=\log _{2} P=\log _{2} n^{n}=n \log _{2} n \approx 26.6 \times 10^{8}=2.66 \times 10^{9} .
$$

$Y$ esta cantidad de información en ardites, cuando es medida en unidades de entropia, apenas significará una contribución de:

$$
2.66 \times 10^{9} \times 10^{-16}=2.66 \times 10^{-7}=0.000000266 .
$$

Sin embargo, el vínculo entre la entropia y la información es indispensable para mantener la consecuencia lógica interna de la teoría de la información. Y, lo que es más importante, existen casos en que la contribución resulta mucho más cuantiosa, incluso medida en unidades de entropia.

\section{Caracteristicas de la anentropia}

En los cambios reversibles se mantiene constante el grado o capacidad de la energía para ejecutar trabajo. Pero en los cambios irreversibles, se produce una degradación o descenso en el grado de la energía. Este grado de la energía se puede expresar con precisión haciéndolo corresponder con la entropia negativa o anentropia. ${ }^{21}$ Entonces, tenemos que la anentropia representa la tendencia de la energía hacia la conservación de su capacidad para producir efectos. Por lo tanto, dentro de un proceso que se mantiene en desequilibrio termodinámico, la anentropia permanece constante. En tanto que, cuando en el proceso se producen cambios de estado que lo aproximan al equilibrio termodinámico, la anentropia decrece. $Y$, cuando se acentúa el desequilibrio termodinámico o la temperatura del proceso disminuye indefinidamente, la anentropia se incrementa. ${ }^{22}$

La anentropia representa así una cualidad activa de la energía. ${ }^{23}$ Un proceso posee anentropia cuando su temperatura no es homogénea, lo mismo

21 Mejor que la palabra hibrida negentropia.

22 Planck, Theory of Heat, pág. 54.

23 Véase, A. M. Yaglom e I. M. Yaglom, Probabilité et Information, París, Dunod; 1959, pág. 60 . 
que cuando su presión no es uniforme o cuando existen en su seno diferencias de potencial. En tal caso, la anentropia puede ser utilizada para ejecutar trabajo, para emitir información o, simplemente, para ser disipada por conducción térmica, resistencia eléctrica, fricción o viscosidad. ${ }^{24}$ Por consiguiente, con la emisión de información se realiza en el proceso una conversión de anentropia en información.

La anentropia es también una medida del orden o de la organización que posee un proceso. ${ }^{25}$ En este sentido, la cualidad distintiva de los procesos orgánicos es su mayor orden. Los organismos vivos absorben continuamente del medio ambiente el orden que necesitan para mantener su vida. En los animales, ese orden es tomado de la anentropia de los compuestos orgánicos más o menos complejos que les sirven de alimento. Después de haber utilizado la anentropia de esos compuestos orgánicos, los animales los devuelven al medio ambiente en forma degradada, pero todavía así contienen anentropia suficiente para ser aprovechada por los vegetales. Por lo tanto, los organismos vivos evitan la muerte abasteciéndose continuamente de anentropia; y su metabolismo consiste propiamente en liberarse de la entropia que producen, adquiriendo en cambio anentropia. ${ }^{26}$

En los organismos vivos, además, el orden de magnitud de la contribución que hacen a la entropia con la información que los mantiene interconectados, si resulta apreciable en comparación con la entropia local. Así, por ejemplo, el número de células que contiene la sangre del organismo humano, es del orden de 25000000000000 . Entonces, la intercomunicación de cada célula con cualquiera otra del propio organismo, es:

$$
P=n^{n} ;
$$

siendo: $n=25000000000000=2.5 \times 10^{13}$.

Por lo tanto, la cantidad de información es:

$$
\begin{gathered}
I=\log _{2} P=\log _{2} n^{n}=n \log _{2} n \approx 45 \times 2.5 \times 10^{13}= \\
=112.5 \times 10^{13}=1.125 \times 10^{13} \text { ardites. }
\end{gathered}
$$

$Y$ esta cantidad de información representa ya una contribución, en unidades de entropia, de:

$$
1.125 \times 10^{15} \times 10^{-16}=1.125 \times 10^{-1}=0.1125 \text {. }
$$

Ahora bien, si consideramos el número total de células que constituyen el organismo humano $o$, todavía más, el número de moléculas que entran

24 Brillouin, op. cit., pág. 116.

25 Véase, N. Wiener, The human use of human beings, Nueva York, Doubleday, 1954, pág. 21.

26 Véase, E. Schroedinger, ¿Qué es la vida?, Buenos Aires, Espasa-Calpe, 1947, págs. 96-108. 
en su composición química, entonces el orden de magnitud de la cantidad de información correspondiente a su interconexión representa valores mucho más apreciables.

En fin, si consideramos el número de átomos que contiene el organismo humano, cuyo orden de magnitud es de 7000000000000000000000000000 , tendremos por consiguiente: $n=7 \times 10^{27}$;

y así, en ardites, la información será de:

$$
I=n \log _{2} n \approx 7 \times 10^{27} \times 107=749 \times 10^{27}=7.49 \times 10^{29} .
$$

$Y$ esta cantidad de información, convertida en unidades de entropia, tiene la considerable magnitud de:

$$
7.49 \times 10^{29} \times 10^{-16}=7.49 \times 10^{13}=74900000000000 .
$$

\section{Certidumbre de la experiencia}

Un cambio que se puede producir o no producir como resultado de una experiencia, es un cambio aleatorio. ${ }^{27}$ La característica principal de los cambios aleatorios es la incertidumbre que existe acerca de su realización. El grado de incertidumbre de una experiencia es la entropia de esa experiencia. Dicha entropia es igual a cero cuando, y sólo cuando, la probabilidad de uno de los resultados posibles sea igual a la unidad, lo cual implica ineludiblemente que todas las demás probabilidades sean nulas. En tal condición, habrá desaparecido la incertidumbre de la experiencia, para convertirse en certidumbre.

Con todo, es indispensable tener en cuenta que al medir la entropia no se consideran todos los factores que determinan conjuntamente su incertidumbre, ni tampoco la manera específica como intervienen. ${ }^{28}$ La entropia depende únicamente de las probabilidades que tienen los diversos resultados posibles de la experiencia, pero su medición es indiferente a la naturaleza de esos resultados. Por lo tanto, la medida del grado de incertidumbre de una experiencia es independiente del significado que puedan tener los posibles resultados. La entropia mide, entonces, el grado de incertidumbre de una manera abstracta.

Esta noción de entropia nos permite medir la influencia que tiene una experiencia sobre otra experiencia, mediante la diferencia de entropia que existe entre ambas. Por eso, la relación entre la entropia y la información es análoga a la relación que existe entre las nociones de potencial y de diferencia de potencial en la física. ${ }^{29}$ La entropia de una experiencia es la información contenida en ella misma, o sea, el máximo de información que

27 Yaglom y Yaglom, op. cit., pág. 5.

28 Yaglom y Yaglom, op. cit., pág. 43.

20 Yaglom y Yaglom, op. cit., pág. 60. 
se puede obtener sobre ella. Lo cual está de acuerdo con lo expresado anteriormente acerca del grado de incertidumbre: mientras mayor sea la incertidumbre de una experiencia, más grande será la información que se pueda obtener de ella al realizarla efectivamente. La cantidad de información adquirida es inversamente proporcional a la entropia y, por ende, directamente proporcional a la anentropia.

\section{Incremento de la entropia}

Es imposible obtener información sobre un proceso sin dejar de provocar cambios en su seno o en el medio que lo rodea. Por ello, al propio tiempo que se adquiere información, se produce un incremento de entropia, ya sea en el proceso mismo o en su vecindad. ${ }^{30}$ En algunos casos, la adquisición de información puede traer consigo un decrecimiento de la entropia del proceso; pero entonces Ia anentropia respectiva tendrá que ser suministrada por algún otro proceso, cuya entropia se incrementará en forma correspondiente. Debido a que la adquisición de información siempre va acompañada de un incremento en la entropia, tenemos que su realización implica un proceso irreversible.

La cantidad de información $I$ que se obtiene en una experiencia, se mide por la diferencia entre la entropia $S_{0}$ que existe inicialmente en el proceso, y la entropia $S_{1}$ que se tiene finalmente, después de realizar la experiencia, o sea:

$$
I=S_{0}-S_{1} \text {. }
$$

Pero esto solamente se cumple en condiciones óptimas, que sólo se pueden suponer idealmente. En general, la cantidad de información obtenida en una experiencia, es menor que el incremento de la entropia. ${ }^{31}$ Por lo tanto, tendremos:

$$
\Delta(S-I) \leq 0 ; \text { y: } \Delta S \leq \Delta I>0 .
$$

Para evitar confusiones, es pertinente especificar que el incremento de entropia $\Delta S$ requerido para obtener información, es independiente del crecimiento que pueda tener la entropia en el mismo proceso o en su vecindad, mientras no se estén ejecutando experiencias. Sin embargo, por lo menos teóricamente, es necesario tomar en cuenta que el costo de entropia que representa la adquisición de información, debe ser despreciable en comparación con la entropia total del proceso observado. Por lo tanto:

$$
\Delta S<<S \text {; ya que: } \Delta I \leq \Delta S<<S ;
$$

30 Brillouin, op. cit., pág. 184 .

31 Brillouin, op. cit., pág. $23^{2}$. 
puesto que la información obtenida siempre es menor que su costo en entropia.

\section{Eficiencia del experimento}

La eficiencia de un experimento se determina mediante la relación establecida entre la cantidad de información obtenida y el incremento de entropia asociado a ella. Esta eficiencia es siempre menor que la unidad, la cual representa su límite máximo inalcanzable. La adquisición de información acerca de un procéso corresponde a un estado de baja entropia o, lo que viene a ser lo mismo, de elevada anentropia. El experimento requiere entonces de fuentes que suministren anentropia, dado que cada observación se ejecuta siempre a expensas de la anentropia del medio circundante. La cantidad mfnima de anentropia requerida en un experimento, es del orden de magnitud de la constante $k$.

La eficiencia $\varepsilon$ de un procedimiento experimental de observación, queda determinada por la relación entre la información obtenida $\Delta I$, a costa de la anentropia $\Delta N$, que es el incremento de entropia $\Delta S$ que acompaña a la ejecución de la observación: ${ }^{32}$

$$
\varepsilon=\frac{\Delta I}{|\Delta N|}=\frac{\Delta I}{\Delta S} \text { ya que: } \mid \Delta N=\Delta S .
$$

La información obtenida en un experimento depende del correspondiente incremento de la entropia, que debe satisfacer la desigualdad:

$$
\Delta S \geqq \Delta I
$$

y, por consiguiente, su eficiencia debe satisfacer la expresión:

$$
\varepsilon=\frac{\Delta I}{\Delta S} \leq 1
$$

Esta relación permite determinar la eficiencia de los diferentes procedimientos de experimentación, lo mismo que coadyuva a establecer su exactitud y su fidedignidad.

\section{Formas de la información}

Hasta aquí nos hemos ocupado de la información únicamente en una de sus formas. En efecto, hemos tratado exclusivamente de la información que se obtiene cuando los casos posibles pueden ser seleccionados vinculándolos 
directamente con los estados físicos del proceso. Por ello, a esa forma de información se la denomina información selectiva. ${ }^{33}$ Pero también es posible adquirir información en una forma más general, como información libre, que es aquella que se obtiene cuando los casos posibles son considerados de manera abstracta, sin precisar en modo alguno su vinculación con los estados físicos del proceso. ${ }^{34}$

La información viva es aquella que se transmite simultáneamente con la energía requerida para su recepción. ${ }^{35}$ Por consiguiente, en la misma operación en que el receptor recibe la información viva, también absorbe la energía con su correspondiente anentropia. En cambio, la información latente es la que es simplemente almacenada o acumulada, por carecer de la energía y la anentropia necesarias para su recepción ulterior, como ocurre con la información impresa, grabada en una cinta magnética o contenida en las perforaciones de una tarjeta. ${ }^{36}$ Entonces, para obtener información, cuando ésta se encuentra en estado latente, se requiere una fuente adicional que suministre la energía y la anentropia necesarias para convertir dicha información latente en información viva.

La información absoluta es la cantidad total de una información determinada, considerándola una sola vez y con independencia del número de ocasiones en que haya sido o pueda ser transmitida y recibida, o bien, transferida y compartida. ${ }^{37}$ La información distribuida es igual al producto de la cantidad de información absoluta, multiplicada por el número de veces que ha sido recibida por transmisión o compartida por transferencia. ${ }^{38}$ Como es fácil advertir, la información distribuida puede aumentar indefinidamente, sin que aumente la información absoluta correspondiente. En todo caso, se tratará de una información nueva para un receptor, cuando éste la recibe por primera vez. En cambio, esa misma información se convertirá en información redundante cuando llegue al mismo receptor por segunda, tercera o enésima vez. ${ }^{39}$

La información relativa o condicional es aquella a la cual se le imponen condiciones, o se le aplican filtros o criterios de selección. Naturalmente, cualquier restricción que se imponga producirá una disminución de la cantidad de información disponible, porque dichas condiciones, criterios o filtros eliminan algunas de las posibilidades del proceso. En consecuencia, la información anticipada que se tenga acerca de un proceso, es enteramente equivalente a la imposición de una condición restrictiva. Por ello, la información

33 Brillouin, op. cit., pág. 291.

34 Brillouin, op. cit., pág. $15^{2}$.

35 Brillouin, op. cit., pág. 259 .

36 Brillouin, op. cit. pág. 261.

37 Brillouin, op. cit., pág. 265 .

38 Brillouin, op. cit., pág. 266.

39 Brillouin, op. cit., pág. 265. 
que se puede obtener cuando ya se cuenta con una información anticipada, es una información relativa o condicional. to $^{4}$

Toda información es información positiva, en el sentido de que su cantidad siempre tiene un valor mayor que cero..1. Sin embargo, puede suceder que, después de haber transmitido una información compuesta de un cierto número de ardites, se requiera obtener su confirmación, la cual puede hacerse mediante un solo ardite, Si o No. En tal caso, la información final también es positiva, teniendo el mismo valor absoluto que el total de la información anterior y también el mismo signo. Pero, igualmente puede ocurrir que, después de haber transmitido una cierta información, se descubra que era errónea o falsa. Entonces, con un nuevo ardite de información es suficiente para anular todos los ardites transmitidos anteriormente. En este sentido, resulta que la información final tiene el mismo valor absoluto que el total de la información anterior, pero con signo contrario, por lo cual tiene que ser considerada como información negativa. ${ }^{42}$

\section{Conversión reciproca entre información y anentropia}

Cuando se obtiene información selectiva sobre un proceso determinado, que no se encuentre aislado, se produce un incremento en la anentropia, a expensas de algún otro proceso. La relación entre el incremento de anentropia y la información selectiva, es:

o bien:

$$
I_{S}=N_{1}-N_{0}
$$

$$
N_{0}=N_{1}-I_{s}
$$

en donde, $I_{S}$ es la información selectiva, $N_{0}$ la anentropia inicial, y $N_{1}$ la anentropia final.

Cuando la información selectiva se adquiere de un proceso que no se encuentra aislado, entonces se produce un decrecimiento de la anentropia:

o, también:

$$
\Delta N_{1} \leq \mathrm{o} ;
$$

y dicho decrecimiento puede ocurrir, en rigor, tanto en $N_{0}$ como en $I_{B}$, o en ambas simultáneamente.

Por otra parte, si consideramos también la información libre $I_{L}$ que se obtiene del mismo proceso, tenemos:

$$
\Delta I_{L} \leq \mathrm{o}, \Delta N_{1} \leq \mathrm{o} ;
$$

40 Brillouin, op. cit., pág. 297.

41 Brillouin, op. cit, pág. 10.

42 Brillouin, op. cit., pág. 10. 
entonces:

$$
\Delta\left(N_{1}+I_{L}\right) \leq 0 ;
$$

y conjugando las dos clases de información, resulta:

$$
\Delta\left(N_{1}+I_{L}\right)=\Delta\left(N_{0}+I_{S}+I_{L}\right)=\Delta\left(N_{0}+I\right) \leq 0 ;
$$

en donde el término $I$ sin índice representa la conjugación de la información libre, $I_{L}$, con la información selectiva, $I_{S} .{ }^{43}$

Tanto la información selectiva como la información libre se obtienen a expensas de la anentropia, ya sea del mismo proceso o de cualquier otro vecino. A su vez, estas dos clases de información se pueden convertir en anentropia. Por consiguiente, tenemos que la información libre y la información selectiva se pueden transformar en anentropia, y viceversa. Además, la suma de información y anentropia se mantiene constante en los cambios reversibles $y$, por lo contrario, decrece en los irreversibles. Pero se trata de una suma en la cual es imposible que alguno de los sumandos pueda quedar reducido a cero. Por consiguiente, la conversión recíproca entre información y anentropia está limitada por la restricción de que la anentropia no se puede transformar totalmente en información, ni tampoco se puede producir la transformación inversa, de información en anentropia, de manera exhaustiva.

\section{Conservación de la cantidad de información}

Las máquinas calculadoras no producen información. Lo único que hacen es someter a ciertos procesos la información que se les suministra como materia prima, para elaborar con ella un producto acabado, pero sin incrementar la información. ${ }^{44}$ En condiciones ideales, la cantidad de información se podrá mantener constante a través del cálculo. Pero, en condiciones normales de realidad, siempre se produce cierta pérdida, de tal manera que la cantidad de información final será menor que la cantidad de información suministrada en un principio. En rigor, la máquina computadora se puede comparar con una red de transmisión, en la cual queda incluida la codificación y el desciframiento. La computadora es alimentada con datos numéricos en la entrada, opera con ellos conforme al programa que también se le suministra y calcula así los datos finales que constituyen el resultado. ${ }^{45}$ Su funcionamiento es también semejante al de una máquina codificadora, que utiliza la información contenida en los datos de la entrada, para descifrarlos en los datos de la salida. Cuando la computadora no comete errores ni hace aproximaciones en el cálculo, la cantidad de información de la salida es igual a la de la

43 Brillouin, op. cit., págs. 153-154.

44 Brillouin, op. cit., pág. 267.

45 Véase, H. Greniewski, Cybernetics without Mathematics, Londres, Pergamon Press, 1960, pág. 21. 
entrada; en caso contrario, se habrá producido una disminución en la cantidad de información.

El proceso que realiza la máquina calculadora se puede comparar todavia mejor con el trabajo de traducción. Al traductor se le suministra un diccionario y las reglas gramaticales que le permiten traducir al español, por ejemplo, un libro escrito originalmente en chino. Este libro en chino representa la entrada; y su traducción representa la salida. La cantidad de información presente en la entrada es, en el caso óptimo, la misma que se puede encontrar presente en la salida; aunque lo más común es que la cantidad de información de la salida sea menor que la de la entrada. ${ }^{46} \mathrm{Si}$ el trabajo del traductor es excelente, el proceso se podrá realizar después en sentido contrario, de manera que sea posible retraducir el libro del español al chino, recuperándose así el texto original o, por lo menos, su equivalente. Las condiciones ideales de operación corresponden a la reversibilidad del proceso y, por consiguiente, a la conservación de la cantidad de información.

Desde el punto de vista estricto de la información, el cálculo no agrega ninguna nueva información, ni tampoco aumenta su cantidad, sino que únicamente la repite en un código diferente. Sin embargo, es enteramente obvio que tanto el cálculo como el desciframiento y la traducción, pueden contribuir a incrementar el valor de uso de la información, haciéndola más significativa para el usuario. Más aún, pueden servir para darle plenamente el significado que después tiene para el usuario, como sucede comúnmente con la traducción que se. hace de una lengua desconocida a una conocida. Pero, en todo caso, es indispensable tener siempre en cuenta que la cantidad de información contenida en los resultados de un cálculo, de un desciframiento o-de una traducción -independientemente de la manera como se hayan realizado - tiene como límite máximo la cantidad de información contenida en los datos iniciales, con el agravante de que dicho límite es generalmente inalcanzable en la práctica.. Esto sucede así porque, de la misma manera como la energía suministrada a un mecanismo cualquiera, siempre es mayor que la cantidad de energía aprovechada útilmente en el funcionamiento del mecanismo, así también la información que se le proporciona a una calculadora de cualquier tipo, siempre es mayor que la cantidad de información contenida en los resultados que elabora.

\section{Transmisión y transferencia de información}

La información es suceptible de comunicarse, ya sea transmitiéndose o transfiriéndose de un proceso a otro $u$ otros procesos. Cuando es transmitida, la información se mantiene en su misma forma. En cambio, cuando es transferida, la información cambia de una forma a otra distinta. La cantidad de

46 Brillouin, op. cit., págs. 295-296. 
información se mide en ardites, cuando se encuentra en la forma de información selectiva. Entonces, la cantidad de información representa una información selectiva medida como información selectiva. ${ }^{47}$ Lo que es más, cuando una información selectiva es transmitida, conserva su forma de información selectiva. Pero, cuando una información selectiva es transferida a otro proceso, entonces se convierte en otra forma distinta de información.

La transferencia de una información selectiva de un proceso a otro, lo cual implica su conversión en otra forma de información, se mide en anentropia. De esta manera, la anentropia representa a la información selectiva medida en su capacidad para convertirse en una magnitud equivalente de otra forma de información. Desde luego, la información selectiva de un proceso se puede transferir a otro proceso en forma de información libre. Igualmente, la información libre de un proceso es susceptible de transferirse a otro proceso, convirtiéndose en información selectiva.

La información viva se transforma en información latente, cuando es almacenada o queda registrada en una memoria. En tal caso, después de que se hace el registro o que se almacena la información, la anentropia excedente es absorbida por otro proceso o se disipa. Por eso, cuando se hace necesario volver a transformar una información latente en información viva, resulta indispensable suministrarle entonces la anentropia requerida para dicha transformación. En consecuencia, también en este caso, queda en claro que la anentropia representa la capacidad que tiene la información latente para convertirse en información viva $y$, recíprocamente, la información viva en información latente.

Como ya lo hemos dicho, la información absoluta es la cantidad total de una información determinada, considerándola una sola vez. Pues bien, esa información puede ser de una sola forma o incluir simultáneamente varias formas distintas. En este último caso, la información absoluta es la suma de las diversas formas de información que la constituyen. Además, esas formas distintas de información son susceptibles de transformarse mutuamente, unas en otras, en magnitudes equivalentes.

Por su parte, la información distribuida representa simplemente una multiplicación de la información, que requiere ineludiblemente un incremento de la anentropia en una magnitud equivalente al incremento de la cantidad de información. En lo que respecta a la información relativa o condicional, la disminución de la cantidad de información se explica por el hecho mismo de que la restricción, la condición, el criterio o el filtro que se imponen entonces, constituyen en rigor una información anticipada, la cual se resta a la cantidad de información disponible. En consecuencia, la información contenida en la restricción, sumada a la información condicional o re-

17 Véase, N. Wiener, Cybernetics, or Control and Communication in the Animal and the Machine, Nueva York, John Wiley, 1948, pág. 111. 
lativa, resulta ser igual a la cantidad absoluta de información existente antes de imponer la restricción.

El problema planteado por la información negativa, se puede interpretar considerándolo desde el punto de vista de la verificación de la información. En tales condiciones, el ardite que sirve para confirmar o para refutar la información transmitida con anterioridad, es una síntesis de ella y, por lo tanto, representa al conjunto entero y tiene el mismo valor en cantidad de información absolua. Aun cuando, para desempeñar esa función de síntesis, se requiere ineludiblemente la transmisión previa de toda la información que sintetiza, ya que únicamente así es como puede ser después confirmada o refutada, empleando para ello un solo ardite de información.

Como conclusión, hemos presentado la analogía existente entre la información y el movimiento. La información selectiva es análoga al movimiento mecánico, en tanto que las otras formas de la información resultan ser análogas a otras de las formas del movimiento. La cantidad de información es análoga a la cantidad de movimiento $\mathrm{y}$, por consiguiente, representa una información selectiva medida como información selectiva. La anentropia es análoga a la energía cinética, ya que representa una información selectiva medida en su capacidad para convertirse en una magnitud equivalente de cualquier otra forma distinta de información. Por último, la información distribuida es análoga al momento de la cantidad de movimiento, porque representa la efectividad de una cantidad de información absoluta determinada. 